\section{Military Technical College Kobry El-Kobbah, Cairo, Egypt}

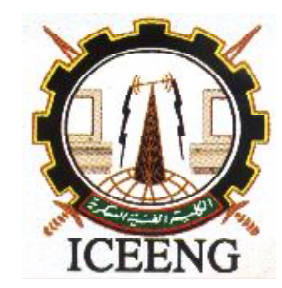

\author{
$10^{\text {th }}$ International Conference \\ on Electrical Engineering \\ ICEENG 2016
}

\title{
Electrical and Optical Characterization of SMOLED and PLED
}

\author{
By \\ Ola Mostafa ${ }^{1}$, Hanady $\mathrm{H}$. \\ Issa $^{2}$, Khaled A. Shehata, ${ }^{3}$ \\ Ahmed El-Bakly ${ }^{4}$
}

\section{Abstract:}

Organic Light-Emitting Diode (OLED) is one of the most interesting areas of scientific research in the field of optoelectronic devices. The aim of this paper is to model the two types of Organic LED which are polymer LEDs (PLED) and OLEDs made with small molecules (SMOLED). The electrical and optical characteristics of the two devices are studied. The simulation tool used is ATLAS-SILVACO. Some parameters are swept in the device to optimize the OLED performance, such as thickness of layers and number of layers. PLED has higher luminance and current density than SMOLED.

\section{Keywords:}

PLED; SMOLED; ITO; MEH-PPV; Alq3

\footnotetext{
${ }^{1}$ Faculty of Engineering, AAST, Cairo, Egypt

${ }^{2}$ Faculty of Engineering, AAST, Cairo, Egypt

${ }^{3}$ Faculty of Engineering, AAST, Cairo, Egypt

${ }^{4}$ Faculty of Engineering, AAST, Cairo, Egypt
} 


\section{Introduction:}

Nowadays Printed Electronics (PE) shows a great potential in realizing large area electronic systems. It could be considered as a competitor to the silicon based technology due do its flexibility, ultra cost effective, lightweight and environmentally friendly [1]. It allows fabricating electronic systems on a flexible and low-cost substrate such as plastic or paper $[2,3]$. The cost of these systems is considerably much lower than the conventional silicon base electronics. On the other hand the cost per function is higher due to the worst resolution of printed electronics [4]. PE opens up new possibilities for many applications and products such as printed RFIDs, organic memories, organic sensors, flexible batteries and organic LED [5].

Recently OLED has drawn the attention for lighting application. The first study of electroluminescence in organic material was in 1950s by Bernanose then by Pope in 1963 and Helfrich in 1965. In 1990 the first OLED was presented by Burroughes which was based on a polymer materials which called Polymer LED (PLED). The main difficulties of OLED and PLED are enhancing its luminance efficiency, increasing its life time and emitting surface [6].

The aim of this paper is to construct and characterize single and double layers of two types of LED, PLEDs and Small Molecules OLEDs (SMOLEDs). The electrical and optical characteristics of the proposed devices are simulated using Atlas tools from Silvaco.

The paper is organized as follows; section II introduces the OLED general structure. Section III explains OLED principle of operation. Section IV shows the devices model and parameters used in the simulations. The simulation results and discussion explained in section V. Finally the conclusion is presented in section VI.

\section{STRUCTURE OF OLED}

OLED has different structures to improve its performance. Fig. 1 shows three structures of the OLED.A one layer device is the basic structure of an OLED. It is made of one organic layer called Emitting Layer (EL) sandwiched between cathode and anode layers. In a two-layer OLED, one organic layer called Hole Transport Layer (HTL)is particularly has the function of transporting holes and the other layer ,Electron Transport Layer (ETL), is intended to transport electrons. Recombination of the holeelectron pair happens at the interface between the two layers, which creates electroluminescence [7]. In three-layer device an extra layer, EL is placed between HTL and ETL. 


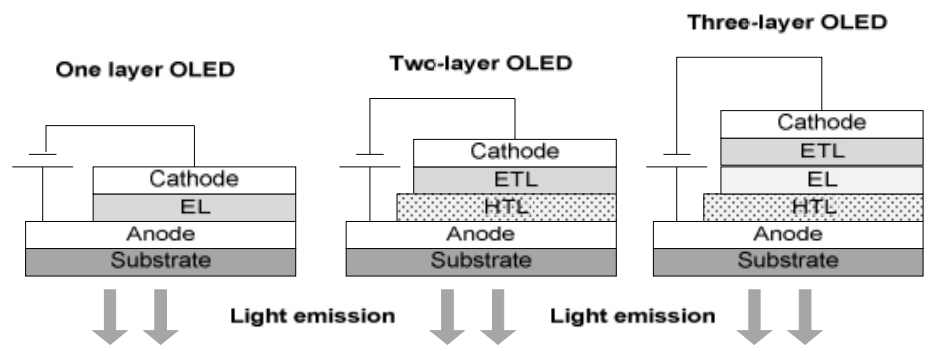

Fig. 1: Different structure of OLEDs

All OLED structures are common in the following layers

- Substrate is a clear plastic, glass or metal foil layer that is a transparent and conductive [1]

- Anode Layer is holes supplying layer of OLED which is connected to the voltage supply. The work function of the anode material ought to be high, so that the energy barrier between the adjacent organic layer and this anode layer is reduced. This will result in effective hole injection to the EL. Indium Tin Oxide (ITO) is the most generally utilized anode material. It has a high transparency (90\%) to visible light, high conductivity and high efficiency. Its work function varies from $4.4 \mathrm{eV}$ to $4.8 \mathrm{eV}$ and its surface properties are basic in the OLED performance $[8,9]$.

- Organic Hole Transport Layer (HTL) only holes from the anode are transported to the EL and blocks electrons from getting away out of the EL on the voltage supply. The HTL principal feature in the OLED is to match the LUMO of the HTL and the energy of the EL or near to the emissive layer's energy gap. The HTL makes the anode layer more conductive according its work function. One of the most widely used materials in HTL is NPB (naphthyl substituted benzidine subsidiary) [10].

- Emissive layer (EL) lies between the HTL and the ETL. It can be a material made of organic molecules or polymers with high quantum efficiency for photoluminescence, colour purity and lifetime [10].

- Organic Electron Transport Layer (ETL) with a suitable electron affinity $(\chi)$, which is a negative energy absorbed or released by an atom or a molecule (X) upon attachment of an electron to form the negative ion (X-). ETL ought to have a high electron drift mobility to transport electrons. (Alq3) and Diphenyl-Phenanthroline (Bphen) are examples of well-known compounds [8].

- Cathode Layer should be a low/medium work function and it varies from $2.9 \mathrm{eV}$ to $4 \mathrm{eV}$, such as lithium, calcium, and magnesium, their use in OLEDs is unreliable especially in ambient atmosphere [8]. 


\section{III.PRINCIPLE OF OPERATION}

To understand the operation of OLED a single layer device is concerned. The energy level diagram of a single layer PLED under forward bias is shown in Fig. 2. When applying a forward bias, electrons are infused from the cathode into the LUMO of the polymer and holes are infused from the anode into the HOMO of the polymer. In this way, the electrons must pass the boundary (electron infusion barrier) between the cathode work function $\varphi$ cathode and the LUMO level of the polymer. Low work function metals, such as, $\mathrm{Ca}, \mathrm{Al}$, or LiF-AL are commonly used to minimize this boundary and give an ohmic contact. A good energy match between cathode and LUMO implies that very little energy is lost when electrons are infused

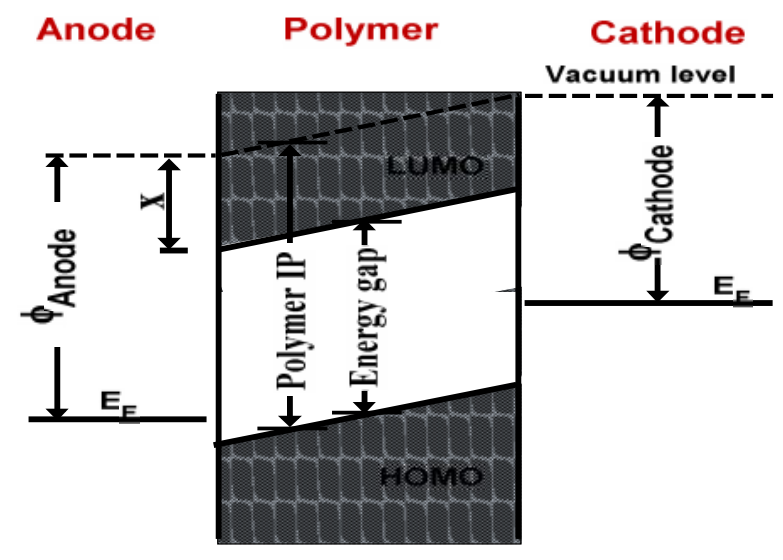

Fig.2: Energy band diagram of PLED device under a forward bias

At the point when an electron and hole reach one another inside the EL, they form a bound excited state called exciton. Depending upon the aggregate spin quantum number of the exciton, it is either a triplet or a singlet. The arrangement proportion of singlet versus triplet is indicated to be 1 versus 3 . The OLEDs using singlet for emission are called fluorescent OLED [11, 12].

The emitted wavelength $\lambda$ depends on the polymer band gap $\mathrm{E}_{\mathrm{g}}$ as follows

$$
\lambda=h \frac{c}{E_{g}}
$$

Where $\mathrm{h}$ is Planck's constant and $\mathrm{C}$ is the speed of light.

\section{DEVICE MODEL AND SIMULATION PARAMETERS}

Two structures of OLEDs are simulated using ATLAS simulator from Silvaco. The simulator is based on the one-dimensional time-independent drift-diffusion transport model, which models the charge carrier transport inside the organic semiconductor. This model contains the continuity equation for electrons and holes and the charge carrier 
motilities for electrons and holes [14, 15]. Poole-Frenkel-like form is the fielddependent mobility model used which is given by [16]

$$
\begin{aligned}
& \mu_{n}(E)=\mu_{n 0} \exp \sqrt{\frac{E}{E_{0}}} \\
& \mu_{p}(E)=\mu_{p 0} \exp \sqrt{\frac{E}{E_{0}}}
\end{aligned}
$$

Where $\mathrm{E}$ is the magnitude of the electric field $\mu_{n 0}$ and $\mu_{p 0}$ are the zero field mobilities and $E_{0}$ is material parameter. The recombination of free electrons and holes is described by Langevin's theory. The Langevin recombination rate is $R_{L}=\frac{q}{\varepsilon}\left(\mu_{n}+\mu_{p}\right) n p$

Where $\varepsilon$ is the permittivity of the organic material, $q$ is the electron charge and $n$ and $p$ are the densities of free electrons and holes respectively.

The simulation parameters implemented in the ATLAS -SILVACO are presented in Table 1.

\begin{tabular}{|c|c|c|c|c|}
\hline Parameter & $\begin{array}{l}\text { MEH } \\
\text { PPV }\end{array}$ & Alq3 & ETL & NPB \\
\hline Electron affinity (eV) & 2.8 & 3.1 & 3.2 & 2.3 \\
\hline Band gap (eV) & 2.1 & 2.7 & 2.6 & 2.9 \\
\hline Relative permittivity & 3 & 3 & 3 & 3 \\
\hline $\begin{array}{c}\text { Hole mobility } \\
\left(\mathrm{cm}^{2} v^{-1} \mathrm{~s}^{-1}\right)\end{array}$ & $0.5 \mathrm{e}-4$ & $0.2 \mathrm{e}-7$ & $0.2 \mathrm{e}-7$ & $0.5 \mathrm{e}-4$ \\
\hline $\begin{array}{c}\text { Electron mobility } \\
\left(\mathrm{cm}^{2} v^{-1} s^{-1}\right)\end{array}$ & $0.5 e-5$ & $0.14 \mathrm{e}-5$ & $0.5 \mathrm{e}-5$ & $0.8 \mathrm{e}-5$ \\
\hline Temperature (K) & 300 & 300 & 300 & 300 \\
\hline Anode work function (ev) & \multicolumn{4}{|c|}{4.8} \\
\hline $\begin{array}{c}\text { Cathode work function } \\
(\mathrm{ev})\end{array}$ & \multicolumn{4}{|c|}{3.6} \\
\hline
\end{tabular}

Table 1: Simulation parameter 


\section{SIMULATION RESULTS AND DISCUSSION}

The electrical and optical properties of both types of OLED are simulated using ATLAS tool. Each type is constructed with two different structures, single layer and double layers structure. The simulation results for each case are explained as following.

\section{Case 1: Single layer devices}

In this case both types of OLED, PLED and SMOLED are constructed with single layer. The electrical (J-V) and optical (power of luminance) are investigated for both types. The first simulation concerns the PLED. It is a one layer device; typically consisting of MEH-PPV polymer as EL which is sandwiched between (ITO) substrate and LiF-AL. MEH-PPV is chosen due to its dissolvability in common organic solvents, in conjunction with a low operating voltage for light emission and relatively high conversion efficiency. The difference between the cathode (Lif-Al) work function $\phi_{L i f-A l}$ and electron affinity $\chi$ of MEH-PPV is $3.6-2.8=0.8 \mathrm{eV}$. This difference considered as a high barrier for electrons injected to MEH-PPV LUMO. The Ionization Potential (IP) of polymer shown in Fig.2 is $4.9 \mathrm{eV}$ and the anode work function $\phi_{\text {ITO }}$ is $4.8 \mathrm{eV}$. Consequently the hole barrier is $4.9-4.8=0.1 \mathrm{eV}$ which is considerably low. The J-V and optical curves of this device with $100 \mathrm{~nm}$ MEH-PPV are shown in Fig. 3.

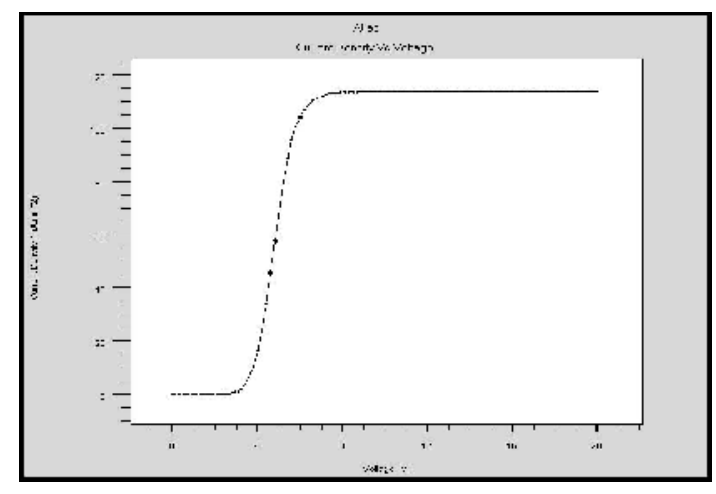

(a)

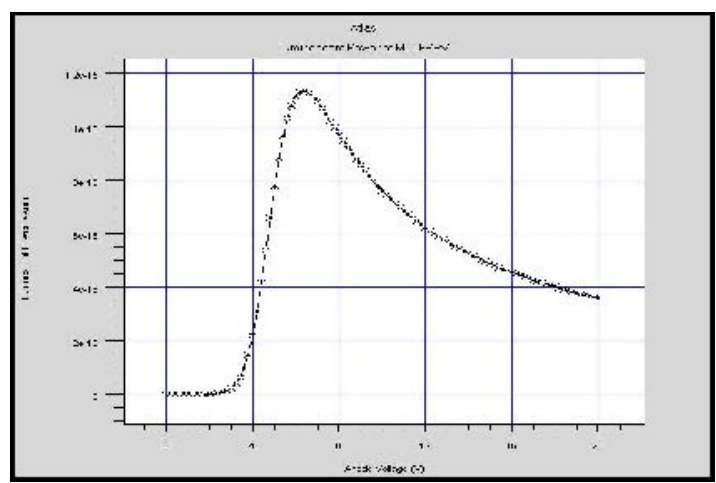

(b)

Fig. 3: simulation results of ITO/MEH-PPV 100nm /LiF-AL,(a) J-V curve, (b) power of luminance

Fig.4.a shows the J-V curves of this device with MEH-PPV thickness varies from 50 $\mathrm{nm}$ to $300 \mathrm{~nm}$. The results show that the current density is inversely proportional to the polymer thickness for high electric fields. In this case, the current density follows the Mott-Gurney law which describes the Space-Charge-Limited Current (SCLC) [18].

The optical property in Fig. 4.b shows that the power luminance of thicker EL is much better than the thinner one. Consequently the device efficiency increases with 
high thickness of EL.

The other structure concerns a single layer SMOLED. It consists of (Alq3) as EL which is sandwiched between ITO as anode and LiF-AL as a cathode. In this structure the anode work function $\phi_{\text {ITO }}$ is $4.8 \mathrm{eV}$ and the IP of ALq3 is $5.8 \mathrm{eV}$. Hence the corresponding hole barrier is $5.8-4.8=1 \mathrm{eV}$.

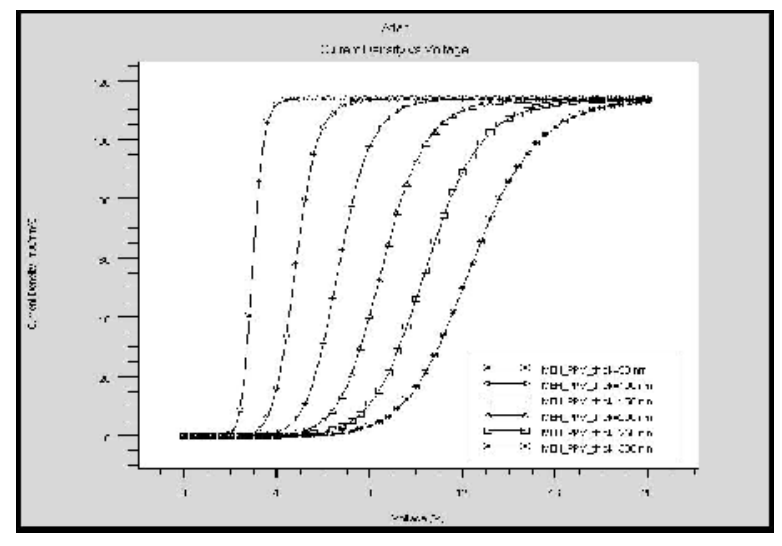

(a)

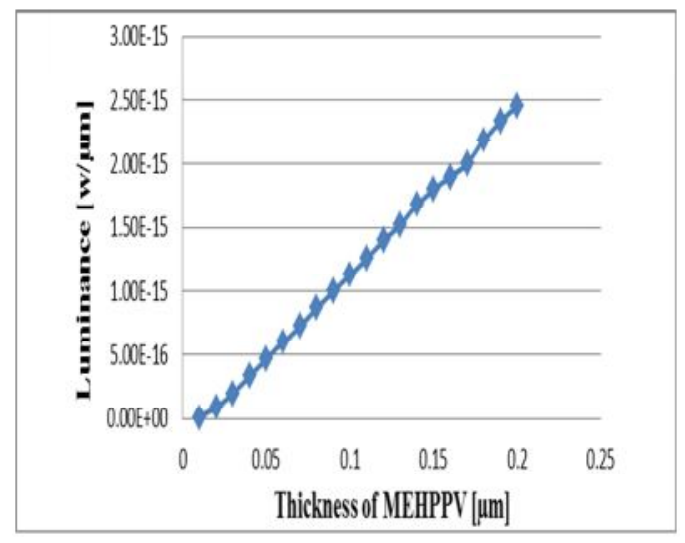

(b)

Fig. 4: (a) current densities, (b) Luminance for ITO/MEH-PPV//Lif-Al with different thicknesses of MEH-PPV

In addition, the cathode work function $\phi_{L i f-A l}$ is $3.6 \mathrm{eV}$ and $\chi$ of Alq3 is $3.1 \mathrm{eV}$, so the electron barrier is $0.5 \mathrm{eV}$. Consequently both electron and hole barriers are considerably high. This result on less number of carriers is being injected and transported therefore less photon emission. The $\mathrm{J}-\mathrm{V}$ and optical curves of this device with $100 \mathrm{~nm}$ of Alq3 are shown in Fig. 5.

In this case the current is limited by injection carriers from electrodes which is defined as Injection Current Limiting (ICL) [18].The model of this current is based on Fowler-Nordheim (FN) tunnelling model. When the applied voltage is sufficient the carriers are injected by tunnelling which limits the device current and hence the photon emission. By increasing the applied voltage the current is limited by the traps. For higher voltage the traps are all filled and the current is limited by space charge. The effect of the EL thickness is examined. Fig. 6 shows that the current density decreases with increasing the thickness of Alq3 but the luminance is increasing with increasing the thickness. 


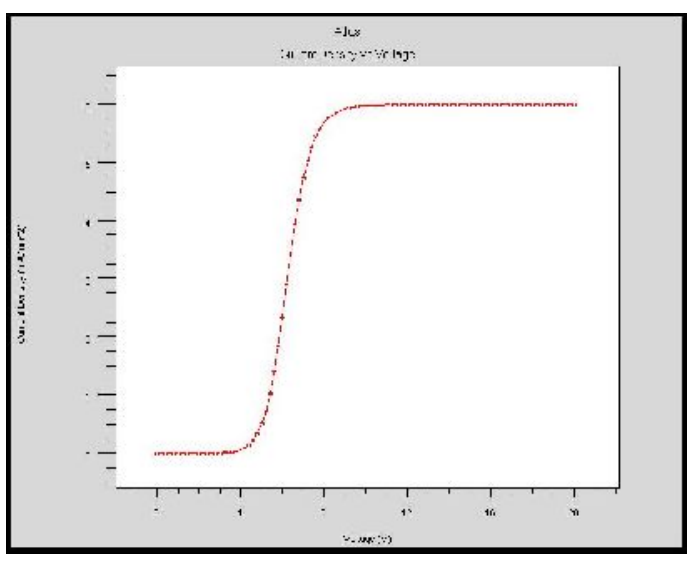

(a)

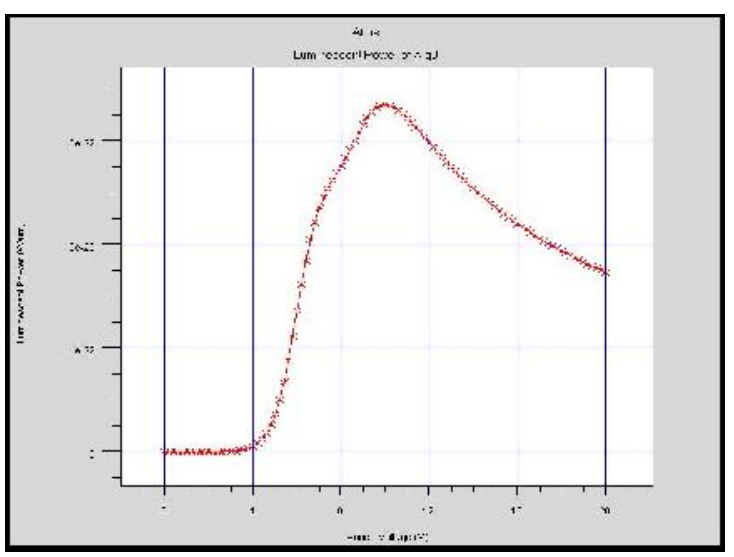

(b)

Fig. 5: (a) simulation results of ITO/ALq3 (100nm)/Lif-AL, (a) J-V curve, (b) power of luminance

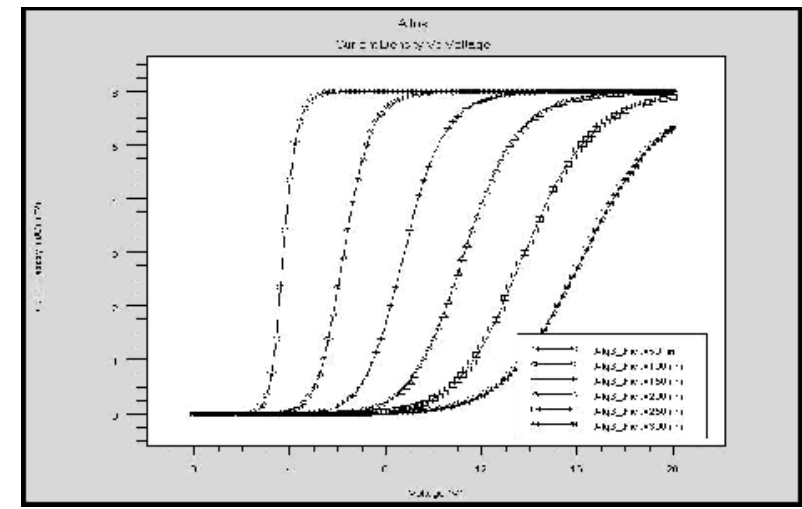

(a)

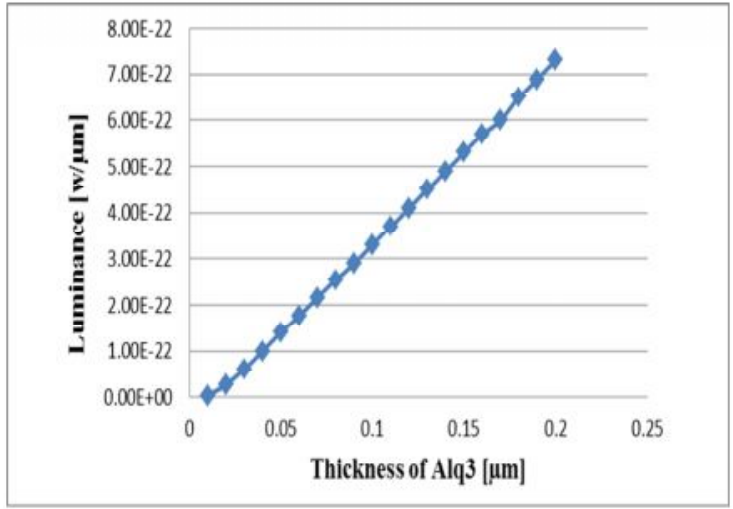

(b)

Fig. 6: (a) current densities (b) Luminance for ITO/ALq3/Lif-Al with different thicknesses of ALq3

\section{Case 2: double layers devices}

In this section of analysis the multilayer devices are analyzed to explore their performance. For PLED device the ETL is added after MEH-PPV. The double layer device structure is; ITO/MEH-PPV/ETL/LiF-AL.

The ETL is chosen to reduce the electron barrier and hence increases the device performance. This is due to the balance between the electron and hole carriers. The J-V and optical curves of this device with $100 \mathrm{~nm}$ MEH-PPV and $100 \mathrm{~nm}$ of ETL are shown in Fig. 7. 


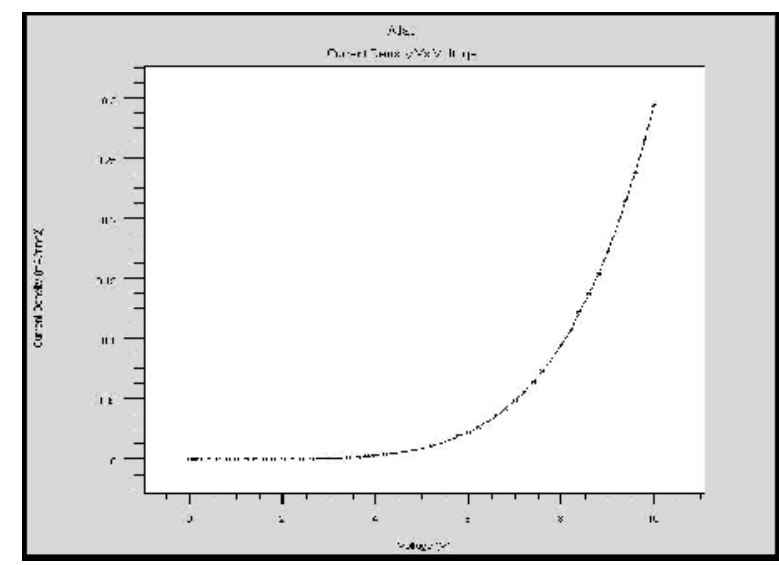

(a)

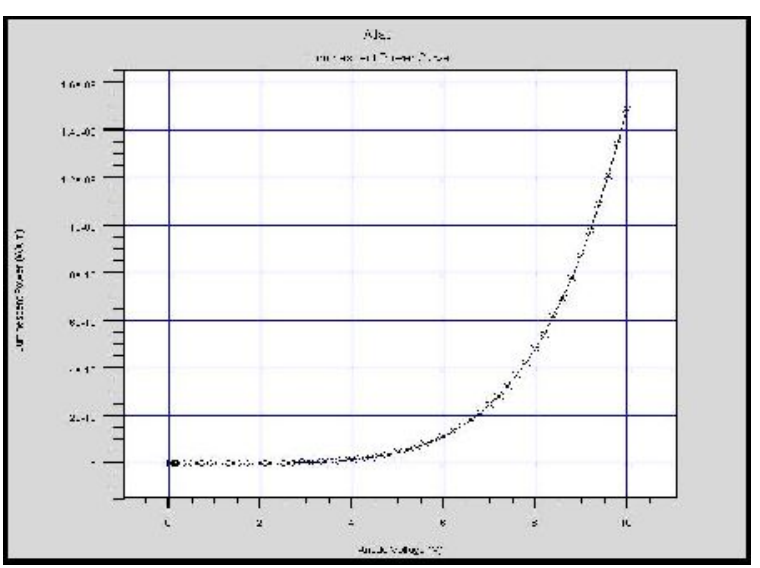

(b)

Fig. 7: Simulation results of ITO/MEH-PPV 100nm/ETL 100nm/Lif-AL, (a) J-V curve, $(b)$ power of luminance

The current density for the device with different thicknesses of ETL and MEH-PPV is as shown in Fig.8.a. The simulation is performed at $10 \mathrm{~V}$ as a forward bias. The simulation results show that for small thickness of MEHPPV and higher ETL thicknesses the current density increases. Moreover the power luminance is also increases which increase the device efficiency as shown in Fig. 8.b.

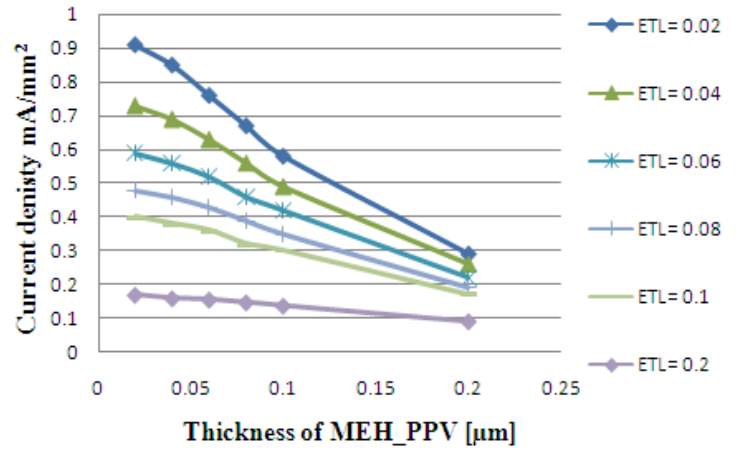

(a)

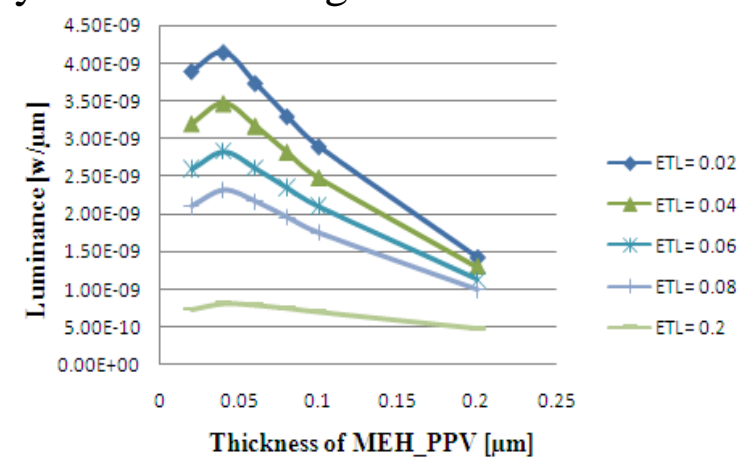

(b)

Fig. 8: (a) Current densities (b) Power of luminance for ITO/MEH-PPV/ETL/LiF-Al with different thicknesses of MEH-PPV and ETL

The last structure is ITO/NPB/Alq3 /LiF-Al. NPB is the most widely used as HTL However, the J-V and optical curves of this device with $100 \mathrm{~nm}$ Alq3 and $100 \mathrm{~nm}$ NPB are shown in Fig. 9. 


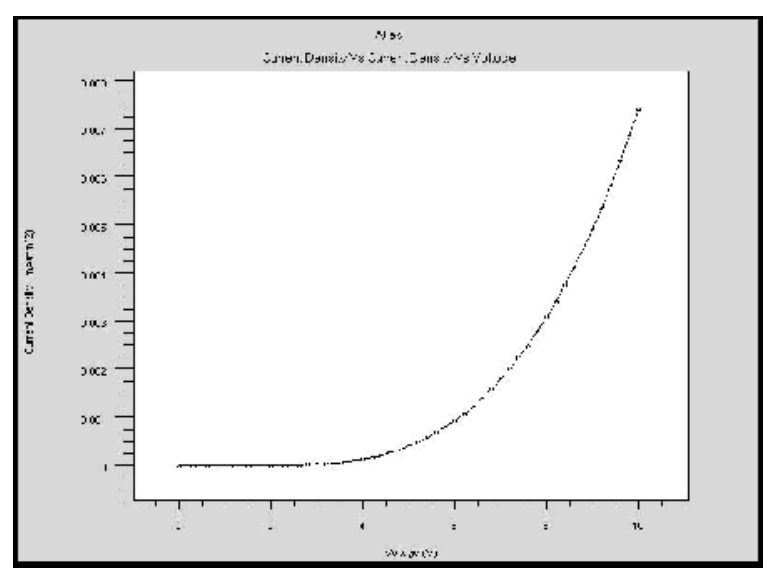

(a)

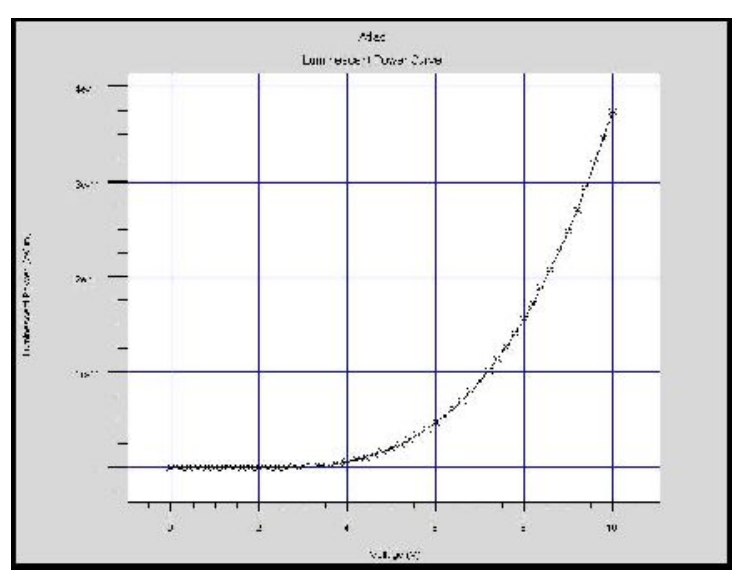

(b)

Fig. 9: Simulation results of ITO/Alq3 100nm/NPB 100nm/Lif-AL, (a) J-V curve, (b) power of luminance

Fig.10 shows the effect of varying thicknesses of NPB and Alq3under forward bias of 10 volt in current density and luminance. The simulation results show that the current density increases as long as the Alq3 and NPB are kept small. This is due to NPB decreases the hole barrier and hence the current increases.

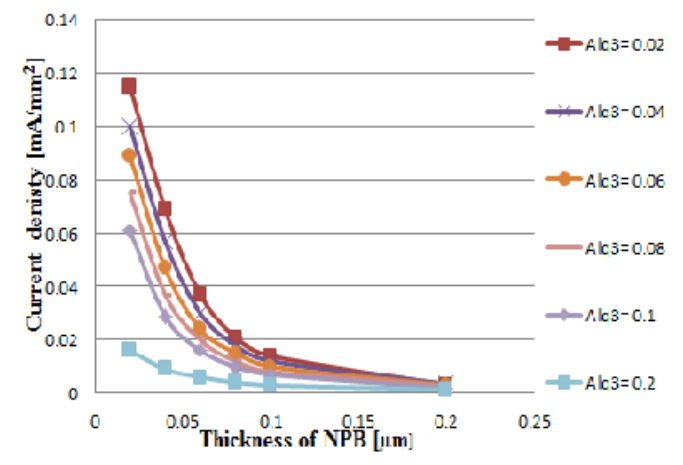

(a)

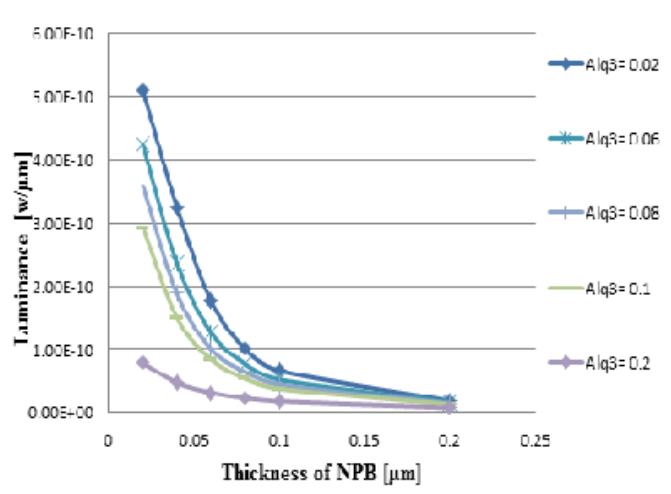

(b)

Fig. 10: (a) current densities (b) power of luminance for ITO/NPB/Alq3 /LiF-Al with different thicknesses of $N P B$ and Alq3

\section{Conclusion}

This paper introduces the two types of OLED, PLED and SMOLED. The optical and current proprieties are simulated using ATLAS from Silvaco. The PLED device is based on MEH-PPV as emissive layer while SMOLED is based on Alq3. The performance of both devices is simulated at different thicknesses of EL. All tests are also performed on two device structures, single and double layers devices. The PLED single layer device shows a higher current density than SMOLED at same forward bias and EL thickness. 
The PLED and SMOLED current densities are $120 \mathrm{~mA} / \mathrm{mm}^{2}$ and $6 \mathrm{~mA} / \mathrm{mm}^{2}$ respectively. The luminance of PLED is $1.2 * 10^{-15} \mathrm{~W} / \mu \mathrm{m}$ while SMOLED is $3 * 10^{-22}$ $\mathrm{W} / \mu \mathrm{m}$. For different EL thicknesses the current density and power of luminance are inversely proportional with EL thickness. Consequently the PLED exhibits better performance than SMOLED. For double-layer PLED, the current density is dramatically reduced and the luminance is improved. The current density is $0.3 \mathrm{~mA} / \mathrm{mm}^{2}$ and the luminance is $1.5^{*} 10^{-9} \mathrm{~W} / \mu \mathrm{m}$ at $10 \mathrm{~V}$ bias and $100 \mathrm{~nm}$ EL. This is due to the energy matching which is provided by ETL between the cathode and EL. Double-layer SMOLED is subjected to same simulation condition. The current density is reduced to be $8 \mu \mathrm{A} / \mathrm{mm}^{2}$ and the luminance is increased to $4^{*} 10^{-11} \mathrm{~W} / \mu \mathrm{m}$. As a result PLED is more stable than SMOLED and has a better performance

\section{$\underline{\text { References }}$}

[1] katsuakiSuganuma, "Introduction to printed electronics", Volume 74, Osaka , Japan , 2014, pp. 6-10.

[2] Taylor \& Francis Group, "Organic light emitting diodes", 6000 Broken Sound Parkway NW, Suite 300, 2012, pp. 20-25.

[3] Challenges of printed electronics on flexible substratesChang, J.; Tong Ge; SanchezSinencio, E.Circuits and Systems (MWSCAS), 2012 IEEE 55th International Midwest Symposium on.

[4]Printed electronics for low-

cost electronic systems: Technology status andapplication development Subramanian, Vivek; Chang, J.B.; de la FuenteVornbrock, A.; Huang, D.C.;Jagannathan, L.; Liao, F.; Mattis, B.; Molesa, S.; Redinger, D.R.; Soltman, D.;Volkman, S.K.; Qintao Zhang Solid-State Circuits Conference, 2008.ESSCIRC 2008. 34th European Year: 2008.

[5]Eugenio Cantatore," Applicationsof organic and printed electronics", 2013.

[6] OLED Technology and Displays SrećkoKunić, ZoranŠego Croatian Radiotelevision, HRT, Prisavlje 3, 10000 Zagreb Croatia,srecko.kunic@hrt.hr, zoran.sego@hrt.hr54th International Symposium ELMAR-2012, , Zadar, Croatia, 12-14 September 2012.

[7] Organic light emitting diodes:Energy saving lighting technology-A review, N.ThejjoKalyani, S.J.Dhoble, renewable and sustainable Energy Reviews 2012.

[8] Graciela Nall, organic electronics, first edition, 2011

[9] A. Layadi, "Sputtered Indium Tin Oxide thin films deposited on glass substrate for photovoltaic application," in International Conference on Renewable Energies and Power Quality, 2010.

[10] Wanli ma, "Water/Methanol soluble conjugated copolymer as an electron transport layer in PLED," advanced materials, 2005.

[11] Simon Rankel," OLEDs Organic Light Emitting Diodes ", Ljubljana, 2004

[12] Yifan Zhang, "Excited State Interactions and Management in Organic Light Emitting Diodes", 2014. 
[13] Electrical and Optical Simulations of a Polymer-Based Phosphorescent Organic Light-Emitting Diode with High Efficiency Mahmoud Al-Sa'di,1 Frank Jaiser,1 Sergey Bagnich,1 Thomas Unger,1James Blakesley,1 Andreas Wilke,2 Dieter Neher11Institute of Physics and Astronomy, Soft Matter Physics, University of Potsdam, KarlLiebknecht-Str. 24-25,D-14476 Potsdam, Germany.

[14] Silvaco, ATLAS user"s manual, Silavaco, Santa Clara, CA.

[15] J. Chan*, A. D. Raki_*, Y. T. Yeow* and A. B. Djuriši_”,Electrical and Optical Simulation of Tris (8-hydroxyquinoline) Aluminium-Based Microcavity Organic Light Emitting Diode (MOLED)", 2005 IEEE.

[16] H. Siemund , F. Bröcker, H. Göbel," Enhancing the electron injection in polymer light-emitting diodes usinga sodium stearate/aluminum bilayer cathode" Organic Electronics 2011.

[17] BOUANATI Sidi Mohammed $\dagger$, N. E. CHABANE SARI, and MOSTEFA KARA Selma, "Electrical and Optical Study of PLED \& OLEDS Structures", 2015.

[18] Zhibin Wang," Design of High Performance Organic Light Emitting Diodes" PhD thesis Graduate Department of Materials Science and Engineering University of Toronto, 2012. 